\title{
Conocimiento sobre la enfermedad renal crónica en jóvenes universitarios cubanos
}

\author{
Delia Leisy Miranda-González, Lesnay Martínez-Rodríguez, Evelyn Fernández-Castillo \\ Departamento Psicología. Universidad Central "Marta Abreu" de las Villas. Cuba
}

Como citar este artículo:

Miranda-González DL, Martínez-Rodríguez L, Fernández-Castillo E. Conocimiento sobre la enfermedad renal crónica en jóvenes universitarios cubanos. Enferm Nefrol. 2020 Jul-Sep;23(3):285-293

\section{Resumen}

Introducción: La alfabetización en salud se reconoce como resultado del proceso de educación para la salud. El conocimiento sobre estilos de vida saludables o las medidas necesarias para evitar el desarrollo o progreso de enfermedades es importante en etapas vulnerables como la juventud.

Objetivo: Evaluar el conocimiento que poseen los jóvenes universitarios cubanos sobre la enfermedad renal crónica.

Material y Método: Estudio observacional descriptivo de corte transversal. El estudio se llevó a cabo en la Universidad Central "Marta Abreu" de las Villas, (Cuba). Se obtuvo una muestra de 420 estudiantes mediante muestreo probabilístico aleatorio estratificado. Se empleó como instrumento el Cuestionario de conocimiento sobre la enfermedad renal crónica. Además se estudiaron otras variables como sexo, edad, carrera, facultad, año académico y provincia de residencia.

Resultados: El 55,7\% de los jóvenes no tenía conocimientos previos sobre la enfermedad renal. No se encontraron diferencias estadísticamente significativas entre los hombres y las mujeres en cuanto nivel de cono-

\section{Correspondencia:}

Lesnay Martínez Rodríguez

Email: lesnay@uclv.cu cimiento por categorías temáticas. Se encontraron diferencias estadísticamente significativas en la puntuación total del Cuestionario entre las diferentes facultades y años académicos.

Conclusiones: Se reconoce un mayor nivel de conocimiento en las categorías temáticas síntomas, características y detección de la enfermedad renal crónica y factores predisponentes, mientras que la categoría de comorbilidades asociadas mostró los peores resultados. Las facultades de Construcciones, Matemática, Física y Computación e Ingeniería Mecánica e Industrial mostraron mejores resultados en las respuestas ofrecidas.

PALABRAS CLAVE: enfermedad renal crónica; alfabetización en salud; joven universitario.

\section{Knowledge about chronic kidney disease in young Cuban university students}

\section{Abstract}

Introduction: Health literacy is recognized as a result of the health education process. Knowledge about healthy lifestyles or measures necessary to avoid the development or progression of diseases is important in vulnerable stages such as youth.

Objective: To evaluate the knowledge that young Cuban university students have about chronic kidney disease. 
Material and Method: Cross-sectional descriptive observational study. The study was carried out at the Universidad Central "Marta Abreu" de las Villas, (Cuba). A sample of 420 students was obtained by stratified random probability sampling. The knowledge questionnaire on chronic kidney disease was used as an instrument. In addition, other variables such as sex, age, university degree, faculty, academic year and province of residence were collected.

Results: $55.7 \%$ of the young people had no previous knowledge about kidney disease. No statistically significant differences were found between men and women in terms of level of knowledge by subject categories. Statistically significant differences were found in the total score of the questionnaire between the different faculties and academic years.

Conclusions: A higher level of knowledge is recognized in the thematic categories focused on symptoms, characteristics and detection of chronic kidney disease and predisposing factors, while the category related to associated comorbidities showed the worst results. The Faculties of Construction, Mathematics, Physics and Computing, and Mechanical and Industrial Engineering showed better results in the answers offered.

KEYWORDS: Chronic kidney disease; health literacy; young university student.

\section{Introducción}

La enfermedad renal es una condición relativamente común, en tanto, una de cada diez personas a nivel mundial la padecen. Por lo general, cursa asintomática y en la gran mayoría de los casos su progreso al estadio 5 pasa inadvertido, por lo que es vital su detección en etapas tempranas ${ }^{1}$. La prevención de la enfermedad renal crónica (ERC) conlleva a retardar la discapacidad, mejorar la calidad de vida del paciente y disminuir la morbimortalidad asociada a ella del mismo modo que evita su evolución, sus complicaciones, así como lograr un tratamiento precoz y adecuado de la misma ${ }^{2,3}$.

La Organización Mundial de la Salud define la alfabetización en salud como "las habilidades sociales y cognitivas que determinan el nivel de motivación y la capacidad de una persona para acceder, entender y utilizar la información de forma que le permita promover y mantener una buena salud" ${ }^{\prime \prime}$. La alfabetización en salud involucra como competencias cruciales: acce- der, entender, evaluar/juzgar y aplicar la información en salud para tomar decisiones hacia el cuidado de la misma, incluyendo no solo al ámbito asistencial, sino también a la prevención de la enfermedad y la promoción de la salud 5 .

De acuerdo con esta definición, la alfabetización en salud supone alcanzar el nivel de conocimientos, habilidades personales y confianza para la asunción de responsabilidad, decisiones concretas, cambio de los estilos y condiciones personales de vida ${ }^{4}$. La baja alfabetización en salud conlleva a una comprensión escasa de la información recibida sobre los tratamientos, pobre conocimiento sobre la cronicidad, detección tardía de la enfermedad, errores en el consumo de medicamentos, inadecuado empleo de los servicios sanitarios, mayor tasa de morbilidad y hospitalizaciones ${ }^{6}$.

Mantener estilos de vida saludables permite desempeñar de manera correcta los retos diarios tanto en contextos educativos como laborales, así cada persona se convierte en el principal responsable de su salud cuidando a diario su cuerpo y mente ${ }^{7}$. Por lo general, los hábitos que se adquieren a temprana edad son los que tienen más probabilidades de mantenerse hasta la edad adulta. La adopción de estilos de vida saludables por parte de los jóvenes, depende en gran medida del conocimiento sobre salud y enfermedad, así como de los factores de riesgo que pueden conllevar al padecimiento de determinadas enfermedades.

Los jóvenes que se encuentran en el contexto universitario se enfrentan a un proceso de transición y por tanto a una etapa vulnerable para desarrollar costumbres y hábitos de riesgo que afectan el proceso de salud-enfermedad. Durante estas etapas los hábitos dietéticos y el ejercicio pueden marcar la diferencia entre una vida sana y el riesgo de sufrir enfermedades en años posteriores ${ }^{8}$. Aunque se reconoce como una población en general sana, teniendo en cuenta que las enfermedades aparecen con mayor frecuencia e intensidad en edades posteriores 9 . A pesar de ello, los jóvenes suelen adoptar estilos de vida que no son saludables y conductas de riesgo que resultan perjudiciales para su salud ${ }^{4}$. El conocimiento de la enfermedad renal desde edades tempranas constituye uno de los elementos más importantes para prevenir y mejorar el pronóstico de padecerla con posterioridad.

Tomando en consideración la importancia que posee el conocimiento sobre la enfermedad, por su elevada prevalencia, morbimortalidad, infradiagnóstico, costos 
económicos y sobre el bienestar de las personas afectadas nos planteamos como objetivo evaluar el conocimiento que poseen los jóvenes universitarios cubanos sobre la enfermedad renal crónica.

\section{Material y métodos}

\section{Diseño}

Estudio observacional descriptivo de corte trasversal.

\section{Contexto de estudio}

El estudio se desarrolló en la sede central de la Universidad Central "Martha Abreu de "Las Villas" (UCLV), ubicada en la provincia de Villa Clara (Cuba).

\section{Muestra}

Se llevó a cabo un muestreo probabilístico aleatorio estratificado por sexo, carrera y año académico durante octubre del 2019 a febrero del 2020. Se estimó la muestra con un error de $5 \%$ y un nivel de confianza de $95 \%$, a partir de una población de 4.395 estudiantes de las 13 facultades de la UCLV, resultando un tamaño muestral de 420 estudiantes de 9 Facultades.

Criterios de inclusión: estudiantes de la UCLV, cubanos $\mathrm{y}$ tener voluntariedad para participar en el estudio y como criterios de exclusión ser estudiantes de postgrado, de otras modalidades de estudio y pertenecientes a otras sedes docentes de la UCLV.

\section{Variables de estudio}

Grado de conocimiento sobre la Enfermedad Renal Crónica.

Otras variables del estudio fueron: sexo, edad, carrera, facultad, año académico y provincia de residencia.

\section{Instrumentos de medida}

- Cuestionario de Conocimientos sobre la Enfermedad Renal Crónica: Instrumento elaborado en España $^{10}$, consta de 28 preguntas o ítems. En el instrumento se exploran en un primer apartado las variables sociodemográficas descritas anteriormente, asimismo, se indaga sobre el conocimiento previo de la enfermedad renal. De acuerdo con las categorías temáticas los ítems se distribuyen en: anatomía y funciones del sistema urinario (1-5), síntomas, características y detección de la ERC (6-13), factores predisponentes a la ERC (14-19), tratamiento de la ERC (20-24) y comorbilidades asociadas a la ERC (25-28). De estas 28 pregun- tas, 24 se responden en formato "verdadero", "falso" o "no sabe o no contesta" ("NS/NC"); y las 4 restantes con opciones de multirrespuesta para 4 opciones a elegir. La calificación del instrumento se realizó mediante el cálculo de las medias, a partir de las opciones de respuesta seleccionadas.

Previo a la aplicación del cuestionario, este fue sometido a criterio de especialistas. Para ello se contó con un total de 8 médicos especialistas en nefrología, con más de 3 años de experiencia y pilotado en 125 estudiantes para detectar y corregir posibles errores en el cuestionario.

\section{Análisis estadístico}

Las variables cualitativas se representaron como una distribución frecuencias absolutas y relativas y las cuantitativas utilizando los valores de la media y la desviación estándar. Para la comparación de 2 muestras se utilizó la prueba T de Student para muestras independientes para explorar las diferencias de medias en cuanto al sexo y las puntuaciones del cuestionario. Para establecer diferencias entre varias medias se realizó un análisis de la varianza (ANOVA) para comparación de medias y la prueba Tukey HSD para el análisis post-hoc. Fueron analizadas también las correlaciones (Pearson) entre la edad de los participantes y las puntuaciones obtenidas. Para el procesamiento de los datos se utilizó el Statistical Package for the Social Sciences (SPSS) versión 21.0 para Windows. Se aceptó significación estadística para $p<0,05$.

\section{Aspectos éticos}

Se obtuvo el consentimiento y la autorización legal de una de las autoras del cuestionario, asumiendo de esta forma las directrices vinculadas al marco legal para este tipo de estudios ${ }^{11}$. Se aplicó el instrumento de forma anónima para garantizar la confidencialidad en las respuestas y una mayor veracidad, respetando en los jóvenes la disposición o no, a responder el mismo. En correspondencia con el Código de Conducta de la Asociación Americana de Psicología ${ }^{12}$ se establecieron como principios generales para la investigación el principio de beneficencia y no maleficencia, fidelidad y responsabilidad, integridad, justicia, respeto por los derechos y dignidad de los participantes.

\section{Resultados}

Se constató un predominio del género femenino representado por el $62,9 \%$ ( $n=264)$. La media de edad fue 
de 20,38 $\pm 1,9$ años. En la Tabla 1 se presenta la muestra según variables sociodemográficas.

Tabla 1. Distribución de la muestra según variables sociodemográficas.

\begin{tabular}{|c|c|c|c|}
\hline & $\begin{array}{c}\text { Variables } \\
\text { sociodemográficas }\end{array}$ & Frecuencia & $\%$ \\
\hline Sexo & Mujeres & 264 & 62,9 \\
\hline Hombres & 156 & 37,1 & \\
\hline \multirow[t]{7}{*}{ Edad } & 17-18 años & 81 & 19,3 \\
\hline & 19-20 años & 145 & 34,5 \\
\hline & 21-22 años & 144 & 34,3 \\
\hline & 23-24 años & 39 & 9,3 \\
\hline & $25-26$ años & 9 & 2,2 \\
\hline & 27-28 años & 1 & 0,2 \\
\hline & 29-30 años & 1 & 0,2 \\
\hline \multirow[t]{5}{*}{ Provincias } & Villa Clara & 224 & 53,3 \\
\hline & Cienfuegos & 50 & 11,9 \\
\hline & Sancti-Spíritus & 78 & 18,6 \\
\hline & Ciego de Ávila & 39 & 9,3 \\
\hline & Camagüey & 29 & 6,9 \\
\hline \multirow[t]{9}{*}{ Facultad } & Ciencias Sociales & 125 & 29,8 \\
\hline & Humanidades & 40 & 9,5 \\
\hline & Matemática, Física y Computación & 40 & 9,5 \\
\hline & Química-farmacia & 30 & 7,1 \\
\hline & Mecánica-Industrial & 35 & 8,3 \\
\hline & Ciencias Económicas & 30 & 7,1 \\
\hline & Ingeniería Eléctrica & 40 & 9,5 \\
\hline & Construcciones & 40 & 9,5 \\
\hline & Ciencias Agropecuarias & 40 & 9,5 \\
\hline \multirow[t]{5}{*}{ Año académico } & $1^{\text {ro }}$ & 130 & 31,0 \\
\hline & $2^{\mathrm{do}}$ & 74 & 17,6 \\
\hline & $3^{\text {ro }}$ & 85 & 20,2 \\
\hline & $4^{\text {to }}$ & 70 & 16,7 \\
\hline & $5^{\text {to }}$ & 61 & 14,5 \\
\hline
\end{tabular}

Fuente: SPSS Versión 22.0
Al analizar la interrogante del cuestionario referida al conocimiento previo de la ERC, se identificaron 183 respuestas positivas $(43,6 \%)$ y 234 respuestas negativas $(55,7 \%)$, respectivamente. $A$ partir del análisis descriptivo por ítems se constataron las respuestas correctas e incorrectas ofrecidas por los jóvenes para cada área temática que evalúa el instrumento (Tabla 2 y Tabla 3 ).

Los resultados de la comparación del grado de conocimiento de los factores del Cuestionario de Conocimientos sobre la Enfermedad Renal Crónica entre las 9 facultades a las que pertenecían los estudiantes, se describen en la Tabla 4.

Las comparaciones post-hoc usando la prueba Tukey HSD indicaron que la puntuación media de los estudiantes pertenecientes a la Facultad Química-Farmacia fue más baja, en la puntuación total del instrumento, que el resto de las facultades agrupadas en los Grupos 2 y 3. Los mejores resultados se dieron en las facultades de Construcciones, Matemática, Física y Computación e Ingeniería Mecánica e Industrial (Tabla 4).

Respecto al año académico de los alumnos, se encontraron también diferencias significativas en el Cuestionario de Conocimientos sobre la Enfermedad Renal Crónica entre los diferentes años académico, presentado los estudiantes pertenecientes al segundo año la puntuación media más baja, y la puntuación más alta los estudiantes pertenecientes a quinto año, como puede observarse en la Tabla 5.

Tabla 2. Análisis de frecuencia de respuestas en preguntas de opción múltiple.

\begin{tabular}{|c|c|c|c|c|}
\hline Preguntas & Opción (a) & Opción (b) & Opción (c) & Opción (d) \\
\hline $\begin{array}{l}\text { 19. La tensión arterial en un paciente } \\
\text { con ERC debe rondar el valor de: }\end{array}$ & $\begin{array}{l}120 / 80 \\
138 / 32,9 \%\end{array}$ & $\begin{array}{l}140 / 80 \\
149 / 35,5 \%\end{array}$ & $\begin{array}{l}140 / 100 \\
106 / 25,2 \%\end{array}$ & $\begin{array}{l}200 / 160 \\
25 / 6,0 \%\end{array}$ \\
\hline $\begin{array}{l}\text { 24. Cuando se tiene ERC hay que controlar } \\
\text { el consumo de alimentos ricos en: }\end{array}$ & $\begin{array}{l}\text { Yodo y Vitamina K } \\
79 / 18,8 \%\end{array}$ & $\begin{array}{l}\text { Hidratos de Carbono, } \\
\text { vitamina E y Grasas } \\
103 / 24,5 \%\end{array}$ & $\begin{array}{l}\text { Fibra vegetal } \\
\text { y Vitamina C } \\
67 / 16,0 \%\end{array}$ & $\begin{array}{l}\text { Sodio, Fosforo, } \\
\text { Potasio y Grasa } \\
170 / 40,5 \%\end{array}$ \\
\hline
\end{tabular}

Leyenda: Respuesta correcta marcada en negrita Fuente: SPSS versión 22.0. 
Tabla 3. Análisis de frecuencia de respuesta en preguntas verdadero_falso_NS/NC.

\begin{tabular}{|c|c|c|c|}
\hline Preguntas & Verdadero & Falso & NS/NC \\
\hline 2. Los riñones segregan hormonas. & $131 / 31,2 \%$ & $204 / 48,6 \%$ & $85 / 20,2 \%$ \\
\hline 3. Los riñones filtran la sangre. & $245 / 58,3 \%$ & $119 / 28,3 \%$ & $56 / 13,3 \%$ \\
\hline $\begin{array}{l}\text { 4. Son los encargados de controlar } \\
\text { la temperatura corporal. }\end{array}$ & $107 / 25,5 \%$ & $191 / 45,5 \%$ & $122 / 29,0 \%$ \\
\hline $\begin{array}{l}\text { 5. Los riñones ayudan a mantener los niveles } \\
\text { de sales de nuestro organismo. }\end{array}$ & $259 / 61,7 \%$ & $49 / 11,7 \%$ & $111 / 26,4 \%$ \\
\hline $\begin{array}{l}\text { 6. Que aparezcan proteínas en la orina } \\
\text { en grandes cantidades es normal, puesto que los riñones } \\
\text { también las filtran. }\end{array}$ & $156 / 37,1 \%$ & $176 / 41,9 \%$ & $88 / 21,0 \%$ \\
\hline 7. Los riñones filtran sustancias de desecho. & $329 / 78,3 \%$ & $53 / 12,6 \%$ & $38 / 9,0 \%$ \\
\hline $\begin{array}{l}\text { 8. La Enfermedad Renal Crónica (ERC) } \\
\text { puede ser asintomática. }\end{array}$ & $122 / 29,0 \%$ & $136 / 32,4 \%$ & $162 / 38,6 \%$ \\
\hline $\begin{array}{l}\text { 9. Si los riñones funcionan mal, } \\
\text { los tobillos se pueden hinchar. }\end{array}$ & $207 / 49,3 \%$ & $77 / 18,3 \%$ & $136 / 32,4 \%$ \\
\hline $\begin{array}{l}\text { 10. Las pruebas para detectar la ERC } \\
\text { son muy complejas y pueden incluso requerir } \\
\text { el ingreso en hospitales. }\end{array}$ & $215 / 51,2 \%$ & $75 / 17,9 \%$ & $130 / 31,0 \%$ \\
\hline $\begin{array}{l}\text { 11. La ERC se puede identificar } \\
\text { en diferentes etapas o estadíos. }\end{array}$ & $271 / 64,5 \%$ & $28 / 6,7 \%$ & $121 / 28,8 \%$ \\
\hline $\begin{array}{l}\text { 13. El gasto para tratar la ERC } \\
\text { es mucho más elevado que el de otras enfermedades crónicas. }\end{array}$ & $163 / 38,8 \%$ & $79 / 18,8 \%$ & $178 / 42,4 \%$ \\
\hline 14. La presión arterial alta puede causar ERC. & $118 / 28.1 \%$ & $117 / 27,9 \%$ & $184 / 43,8 \%$ \\
\hline 15. La diabetes es un factor de riesgo para padecer ERC. & $246 / 58,6 \%$ & $47 / 11,2 \%$ & $127 / 30,2 \%$ \\
\hline 16. El consumo de tabaco aumenta el riesgo de padecer ERC. & $220 / 52,4 \%$ & $90 / 21,4 \%$ & $110 / 26,2 \%$ \\
\hline $\begin{array}{l}\text { 17. Las personas jóvenes tienen el mismo riesgo } \\
\text { de padecer ERC que las de edad más avanzada. }\end{array}$ & $177 / 42,1 \%$ & $150 / 35,7 \%$ & $93 / 22,1 \%$ \\
\hline 18. La obesidad predispone a padecer ERC. & $229 / 54,5 \%$ & $62 / 14,8 \%$ & $129 / 30,7 \%$ \\
\hline $\begin{array}{l}\text { 20. La diálisis se utiliza para suplir la función } \\
\text { de filtrado del riñón. }\end{array}$ & $330 / 78,6 \%$ & $20 / 4,8 \%$ & $69 / 16,4 \%$ \\
\hline $\begin{array}{l}\text { 21. Puedo tomar Ibuprofeno para combatir el dolor } \\
\text { cuando padezco ERC. }\end{array}$ & $111 / 26,4 \%$ & $141 / 33,6 \%$ & $168 / 40,0 \%$ \\
\hline $\begin{array}{l}\text { 22. Respecto a la diálisis. Existe alguna alternativa } \\
\text { al tratamiento hospitalario. }\end{array}$ & $152 / 36,2 \%$ & $98 / 23,3 \%$ & $170 / 40,5 \%$ \\
\hline $\begin{array}{l}\text { 23. Una vez instaurada la ERC necesitará un tratamiento } \\
\text { de por vida. }\end{array}$ & $259 / 61,7 \%$ & $64 / 15,2 \%$ & $97 / 23,1 \%$ \\
\hline 25. La anemia es una complicación común de la ERC. & $166 / 39,5 \%$ & $77 / 18,3 \%$ & $177 / 42,1 \%$ \\
\hline 26. Las enfermedades óseas son complicaciones comunes de la ERC & $91 / 21,7 \%$ & $123 / 29,3 \%$ & $206 / 49,0 \%$ \\
\hline 27. El riesgo de sufrir un infarto está relacionado con la ERC. & $96 / 22,9 \%$ & $169 / 40,2 \%$ & $155 / 36,9 \%$ \\
\hline $\begin{array}{l}\text { 28. La ERC aumenta el riesgo de morir si se padece } \\
\text { cualquier otra enfermedad grave. }\end{array}$ & $289 / 68,8 \%$ & $28 / 6,7 \%$ & $103 / 24,5 \%$ \\
\hline
\end{tabular}

Leyenda: Respuesta correcta marcada en negrita Fuente: SPSS versión 22.0.

No se encontraron diferencias estadísticamente significativas entre hombres y mujeres, respecto en los ítems del Cuestionario de conocimientos sobre la Enfermedad Renal Crónica.
El análisis correlacional entre la variable edad y las categorías temáticas se muestran en la Tabla 6, donde podemos apreciar que no se alcanza la significación estadística en ninguna de las áreas temáticas recogidas en el cuestionario. 
Tabla 4. Comparaciones entre las facultades a las pertenecen los estudiantes y los factores del Cuestionario de Conocimientos sobre la Enfermedad Renal Crónica.

\begin{tabular}{|c|c|c|c|c|c|c|c|c|c|c|c|c|c|}
\hline & CS & H & MFC & QF & MI & CE & IE & C & CA & \multirow[b]{2}{*}{$\mathbf{F}$} & \multirow[b]{2}{*}{$\boldsymbol{p}$} & \multirow{2}{*}{$\Pi_{2}$} & \multirow{2}{*}{$\begin{array}{l}\text { Comparaciones } \\
\text { Post Hoc }\end{array}$} \\
\hline & M(SD) & M(SD) & M(SD) & M(SD) & M(SD) & M(SD) & M(SD) & M(SD) & M(SD) & & & & \\
\hline $\begin{array}{l}\text { Anatomía } \\
\text { y funciones } \\
\text { del sistema urinario }\end{array}$ & $\begin{array}{l}10,47 \\
(2.37)\end{array}$ & $\begin{array}{c}9,87 \\
(1.64)\end{array}$ & $\begin{array}{l}10,72 \\
(2,27)\end{array}$ & $\begin{array}{c}9,20 \\
(1,84)\end{array}$ & $\begin{array}{l}10,42 \\
(2,00)\end{array}$ & $\begin{array}{c}9,73 \\
(1,72)\end{array}$ & $\begin{array}{l}10,20 \\
(2,15)\end{array}$ & $\begin{array}{l}10,25 \\
(2,19)\end{array}$ & $\begin{array}{c}9,20 \\
(1,45)\end{array}$ & 2,902 &, 004 &, 053 & $\begin{array}{l}\mathrm{Gl}: \mathrm{QF}=\mathrm{CA} \\
\mathrm{G} 2: \mathrm{CE}<\mathrm{H}<\mathrm{IE}<\mathrm{C}<\mathrm{MI}<\mathrm{CS}<\mathrm{MFC} \\
\mathbf{G 1}<\mathrm{G} 2\end{array}$ \\
\hline $\begin{array}{l}\text { Síntomas } \\
\text { y características } \\
\text { de la ERC }\end{array}$ & $\begin{array}{c}14,7 \\
(3,33)\end{array}$ & $\begin{array}{c}14,4 \\
(2,59)\end{array}$ & $\begin{array}{c}16,0 \\
(3,99)\end{array}$ & $\begin{array}{l}13,26 \\
(1,98)\end{array}$ & $\begin{array}{l}16,25 \\
(2,98)\end{array}$ & $\begin{array}{l}14,76 \\
(2,82)\end{array}$ & $\begin{array}{l}15,18 \\
(2,77)\end{array}$ & $\begin{array}{l}15,92 \\
(3,61)\end{array}$ & $\begin{array}{l}13,55 \\
(2,72)\end{array}$ & 4,168 &, 000 & ,075 & $\begin{array}{l}\mathrm{Gl}: \mathrm{QF}=\mathrm{CA} \\
\mathrm{G} 2: \mathrm{CA}<\mathrm{CE}<\mathrm{H}<\mathrm{IE}<\mathrm{C}<\mathrm{MI}<\mathrm{CS}<\mathrm{MFC} \\
\mathbf{G l}<\mathrm{G} 2\end{array}$ \\
\hline $\begin{array}{l}\text { Factores } \\
\text { predisponentes } \\
\text { de la ERC }\end{array}$ & $\begin{array}{l}11,29 \\
(2,69)\end{array}$ & $\begin{array}{l}10,92 \\
(2,59)\end{array}$ & $\begin{array}{l}11,30 \\
(2,96)\end{array}$ & $\begin{array}{c}9,83 \\
(1,80)\end{array}$ & $\begin{array}{l}11,42 \\
(2,71)\end{array}$ & $\begin{array}{l}10,86 \\
(2,86)\end{array}$ & $\begin{array}{l}10,69 \\
(2,88)\end{array}$ & $\begin{array}{l}12,23 \\
(3,12)\end{array}$ & $\begin{array}{l}10,67 \\
(2,49)\end{array}$ & 2,224 &, 025 & ,041 & $\begin{array}{l}\mathrm{Gl}: \mathrm{QF} \\
\mathrm{G} 2: \mathrm{CA}<\mathrm{CE}<\mathrm{H}<\mathrm{IE}<\mathrm{C}<\mathrm{MI}<\mathrm{CS}<\mathrm{MFC} \\
\mathbf{G l}<\mathrm{G} 2\end{array}$ \\
\hline $\begin{array}{l}\text { Tratamiento } \\
\text { de la ERC }\end{array}$ & $\begin{array}{l}10,23 \\
(2,27)\end{array}$ & $\begin{array}{c}9,62 \\
(2,27)\end{array}$ & $\begin{array}{l}10,12 \\
(2,51)\end{array}$ & $\begin{array}{c}8,03 \\
(1,51)\end{array}$ & $\begin{array}{c}9,25 \\
(2,11)\end{array}$ & $\begin{array}{c}9,33 \\
(2,20)\end{array}$ & $\begin{array}{l}10,89 \\
(2,18)\end{array}$ & $\begin{array}{l}10,45 \\
(2,52)\end{array}$ & $\begin{array}{l}10,42 \\
(2,01)\end{array}$ & 5,299 &, 000 &, 093 & $\begin{array}{l}\mathrm{Gl}: \mathrm{QF} \\
\mathrm{G} 2: \mathrm{MI}<\mathrm{CE} \\
\mathrm{G} 3: \mathrm{H}<\mathrm{MFC}<\mathrm{CS}<\mathrm{CA}<\mathrm{C}<\mathrm{IE} \\
\mathrm{Gl}<\mathrm{G} 2<\mathrm{G} 3\end{array}$ \\
\hline $\begin{array}{l}\text { Comorbilidades } \\
\text { asociadas }\end{array}$ & $\begin{array}{c}8,32 \\
(2,19)\end{array}$ & $\begin{array}{c}7,82 \\
(1,95)\end{array}$ & $\begin{array}{c}8,55 \\
(2,43)\end{array}$ & $\begin{array}{c}5,83 \\
(1,08)\end{array}$ & $\begin{array}{c}7,94 \\
(1,81)\end{array}$ & $\begin{array}{c}7,30 \\
(1,91)\end{array}$ & $\begin{array}{c}8,22 \\
(2,20)\end{array}$ & $\begin{array}{c}9,15 \\
(2,48)\end{array}$ & $\begin{array}{c}7,40 \\
(2,09)\end{array}$ & 7,05 &, 000 & ,012 & $\begin{array}{l}\mathrm{Gl}: \mathrm{QF} \\
\mathrm{G} 2: \mathrm{CE}<\mathrm{CA} \\
\mathrm{G} 3: \mathrm{H}<\mathrm{MI}<\mathrm{IE}<\mathrm{MFC}<\mathrm{C} \\
\mathrm{Gl}<\mathrm{G} 2<\mathrm{G3}\end{array}$ \\
\hline Puntuación Total & $\begin{array}{l}55,06 \\
(9,63)\end{array}$ & $\begin{array}{l}52,64 \\
(6,05)\end{array}$ & $\begin{array}{c}56,72 \\
(11,47)\end{array}$ & $\begin{array}{l}46,16 \\
(4,99)\end{array}$ & $\begin{array}{l}55,31 \\
(8,09)\end{array}$ & $\begin{array}{l}52,00 \\
(6,92)\end{array}$ & $\begin{array}{l}55,24 \\
(7,73)\end{array}$ & $\begin{array}{l}58,30 \\
(9,89)\end{array}$ & $\begin{array}{l}51,25 \\
(7,11)\end{array}$ & 6,060 &, 000 & ,106 & $\begin{array}{l}\mathrm{G} 1: \mathrm{QF} \\
\mathrm{G} 2: \mathrm{CA}<\mathrm{CE} \\
\mathrm{G} 3: \mathrm{H}<\mathrm{CS}<\mathrm{IE}<\mathrm{MI}<\mathrm{MFC}<\mathrm{C} \\
\mathbf{G 1}<\mathrm{G} 2<\mathrm{G} 3\end{array}$ \\
\hline
\end{tabular}

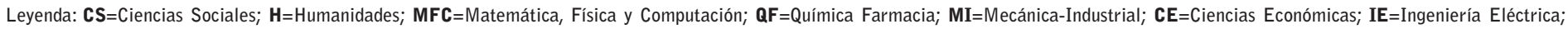
$\mathbf{C}=$ Construcciones; $\mathbf{C A}=$ Ciencias Agropecuarias.

Tabla 5. Comparaciones entre los años académicos y los factores del Cuestionario de conocimientos sobre la Enfermedad Renal Crónica.

\begin{tabular}{|c|c|c|c|c|c|c|c|c|c|}
\hline & lro & $2 \mathrm{do}$ & 3ro & 4to & 5to & & & $\Pi_{2}$ & Comparaciones \\
\hline & $M(S D)$ & $M(S D)$ & $\mathrm{M}(\mathrm{SD})$ & $M(S D)$ & $M(S D)$ & $\mathrm{F}$ & $p$ & partial & Post Hoc \\
\hline $\begin{array}{l}\text { Anatomía y funciones } \\
\text { del sistema urinario }\end{array}$ & 10,37 & $\begin{array}{c}9,58 \\
(2,21)\end{array}$ & $\begin{array}{l}10,02 \\
(2,25)\end{array}$ & $\begin{array}{l}10,00 \\
(1,93)\end{array}$ & $\begin{array}{l}10,52 \\
(1,91)\end{array}$ & $\begin{array}{l}2,370 \\
(2,09)\end{array}$ &, 052 &, 002 & $\begin{array}{l}\mathrm{Gl}: 2 \text { do } \\
\mathrm{G} 2: 4 \text { to }<3 \text { ro } 0 \text { lro } 0<5 \text { to } \\
\mathbf{G 1}<\mathbf{G 2}\end{array}$ \\
\hline Síntomas y características de la ERC & $\begin{array}{c}15,4 \\
(3,67)\end{array}$ & $\begin{array}{l}14,14 \\
(2,78)\end{array}$ & $\begin{array}{l}14,62 \\
(2,99)\end{array}$ & $\begin{array}{l}14,60 \\
(2,60)\end{array}$ & $\begin{array}{l}15,45 \\
(3,45)\end{array}$ & 2,539 &, 039 &, 002 & $\mathrm{Gl}: 2 \mathrm{~d} 0<4$ to $<3$ ro $<1$ ro $<5$ to \\
\hline Factores predisponentes de la ERC & $\begin{array}{l}11,47 \\
(2,74)\end{array}$ & $\begin{array}{l}10,37 \\
(2,40)\end{array}$ & $\begin{array}{l}11,41 \\
(2,48)\end{array}$ & $\begin{array}{l}10,80 \\
(2,79)\end{array}$ & $\begin{array}{l}11,80 \\
(2,95)\end{array}$ & 2,224 & 010 & 031 & $\begin{array}{l}\text { G1:2do } \\
\text { G2:4to<3ro<l ro }<5 \text { to } \\
\mathbf{G 1}<\mathbf{G 2}\end{array}$ \\
\hline Tratamiento de la ERC & $\begin{array}{l}10,06 \\
(2,33)\end{array}$ & $\begin{array}{c}9,91 \\
(2,08)\end{array}$ & $\begin{array}{c}9,77 \\
(2,46)\end{array}$ & $\begin{array}{c}9,87 \\
(2,11)\end{array}$ & $\begin{array}{l}10,16 \\
(2,49)\end{array}$ & ,347 & ,846 &, 003 & $\mathrm{Gl}: 3$ ro $<4$ to $<2$ do $<1$ r $0<5$ to \\
\hline Comorbilidades asociadas & $\begin{array}{c}8,26 \\
(2,24)\end{array}$ & $\begin{array}{c}7,54 \\
(2,24)\end{array}$ & $\begin{array}{c}7,88 \\
(2,15)\end{array}$ & $\begin{array}{c}7,94 \\
(2,33)\end{array}$ & $\begin{array}{c}8,19 \\
(2,13)\end{array}$ & 1,454 & ,216 & 013 & $\mathrm{Gl}: 2 \mathrm{~d} 0<3$ ro $<4$ to $<5$ to $<1$ ro \\
\hline Puntuación Total & $\begin{array}{l}55,58 \\
(9,72)\end{array}$ & $\begin{array}{l}51,41 \\
(8,03)\end{array}$ & $\begin{array}{l}53,82 \\
(8,66)\end{array}$ & $\begin{array}{l}53,00 \\
(7,93)\end{array}$ & $\begin{array}{l}55,88 \\
(, 64)\end{array}$ & 3,416 &, 009 &, 032 & $\begin{array}{l}\mathrm{Gl}: 2 \text { do } \\
\mathrm{G} 2: 4 \text { to }<3 \text { ro }<1 \text { ro } 0<5 \text { to } \\
\mathbf{G 1}<\mathbf{G 2}\end{array}$ \\
\hline
\end{tabular}


Tabla 6. Correlaciones entre la edad de los participantes y los factores del Cuestionario de Conocimientos sobre la Enfermedad Renal Crónica.

\begin{tabular}{|c|c|c|c|c|c|c|c|c|}
\hline & & $\begin{array}{c}\text { Edad } \\
\text { del } \\
\text { participante }\end{array}$ & $\begin{array}{l}\text { Anatomía } \\
\text { y funciones } \\
\text { del sistema } \\
\text { urinario }\end{array}$ & $\begin{array}{l}\text { Síntomas y } \\
\text { características } \\
\text { de la ERC }\end{array}$ & $\begin{array}{l}\text { Factores } \\
\text { predisponentes } \\
\text { de la ERC }\end{array}$ & $\begin{array}{l}\text { Tratamiento } \\
\text { de la ERC }\end{array}$ & $\begin{array}{l}\text { Comorbilidades } \\
\text { asociadas }\end{array}$ & $\begin{array}{l}\text { Puntuación } \\
\text { Total }\end{array}$ \\
\hline $\begin{array}{l}\text { Edad del } \\
\text { participante }\end{array}$ & $\begin{array}{c}\text { Correlación de Pearson } \\
\text { Sig. (unilateral) } \\
\text { N }\end{array}$ & $\begin{array}{c}1 \\
420\end{array}$ & & & & & & \\
\hline $\begin{array}{l}\text { Anatomía } \\
\text { y funciones } \\
\text { del sistema } \\
\text { urinario }\end{array}$ & $\begin{array}{c}\text { Correlación de Pearson } \\
\text { Sig. (unilateral) } \\
\text { N }\end{array}$ & $\begin{array}{l}, 023 \\
, 318 \\
419\end{array}$ & $\begin{array}{c}1 \\
419\end{array}$ & & & & & \\
\hline $\begin{array}{l}\text { Síntomas y } \\
\text { características } \\
\text { de la ERC }\end{array}$ & $\begin{array}{c}\text { Correlación de Pearson } \\
\text { Sig. (unilateral) } \\
\text { N }\end{array}$ & $\begin{array}{l}-, 024 \\
, 311 \\
417\end{array}$ & $\begin{array}{c}388(* *) \\
, 000 \\
417\end{array}$ & $\begin{array}{c}1 \\
417\end{array}$ & & & & \\
\hline $\begin{array}{l}\text { Factores } \\
\text { predisponentes } \\
\text { de la ERC }\end{array}$ & $\begin{array}{c}\text { Correlación de Pearson } \\
\text { Sig. (unilateral) } \\
\text { N }\end{array}$ & $\begin{array}{l}, 007 \\
444 \\
417\end{array}$ & $\begin{array}{c}, 356(* *) \\
, 000 \\
417\end{array}$ & $\begin{array}{c}, 458(* *) \\
, 000 \\
416\end{array}$ & $\begin{array}{c}1 \\
417\end{array}$ & & & \\
\hline $\begin{array}{l}\text { Tratamiento } \\
\text { de la ERC }\end{array}$ & $\begin{array}{c}\text { Correlación de Pearson } \\
\text { Sig. (unilateral) } \\
\text { N }\end{array}$ & $\begin{array}{l}-, 037 \\
, 226 \\
419\end{array}$ & $\begin{array}{c}227(* *) \\
, 000 \\
418\end{array}$ & $\begin{array}{c}351(* *) \\
, 000 \\
416\end{array}$ & $\begin{array}{c}308(* *) \\
, 000 \\
416\end{array}$ & $\begin{array}{c}1 \\
419\end{array}$ & & \\
\hline $\begin{array}{l}\text { Comorbilidades } \\
\text { asociadas }\end{array}$ & $\begin{array}{c}\text { Correlación de Pearson } \\
\text { Sig. (unilateral) } \\
\text { N }\end{array}$ & $\begin{array}{l}-, 037 \\
, 223 \\
420\end{array}$ & $\begin{array}{c}413(* *) \\
, 000 \\
419\end{array}$ & $\begin{array}{c}509(* *) \\
, 000 \\
417\end{array}$ & $\begin{array}{c}470(* *) \\
, 000 \\
417\end{array}$ & $\begin{array}{c}481\left(^{* *}\right) \\
, 000 \\
419\end{array}$ & $\begin{array}{c}1 \\
420\end{array}$ & \\
\hline Puntuación Total & $\begin{array}{l}\text { Correlación de Pearson } \\
\text { Sig. (unilateral) } \\
\text { N }\end{array}$ & $\begin{array}{r}-, 031 \\
, 264 \\
415\end{array}$ & $\begin{array}{c}, 626\left(^{* *}\right) \\
, 000 \\
415\end{array}$ & $\begin{array}{c}, 796\left(^{* *}\right) \\
, 000 \\
415\end{array}$ & $\begin{array}{c}, 737(* *) \\
, 000 \\
415\end{array}$ & $\begin{array}{c}, 632\left(^{* *}\right) \\
, 000 \\
415\end{array}$ & $\begin{array}{c}, 779(* *) \\
, 000 \\
415\end{array}$ & $\begin{array}{c}1 \\
415\end{array}$ \\
\hline
\end{tabular}

** La correlación es significante al nivel 0,01 (unilateral).

\section{Discusión}

Los resultados del presente estudio muestran que existe un conocimiento deficiente de la enfermedad renal en jóvenes universitarios cubanos. Se evidenció un mejor conocimiento acerca de los síntomas, características y detección de la enfermedad, siendo los aspectos menos conocidos los relativos a las comorbilidades asociadas a dicha patología. La falta de conocimiento entre los jóvenes no constituye en sí mismo un resultado sorprendente, dado que también se observa esta falta de conocimiento en poblaciones que ya presentan enfermedades renales ${ }^{13-16}$ o relacionadas con la mis$\mathrm{ma}^{17,18}$.

Existe evidencia de numerosos estudios que demuestran el bajo nivel de conocimientos existentes en temas referidos a la alfabetización en salud sobre diversas enfermedades ${ }^{19-22}$. Los datos que abordó nuestro estudio coinciden con los resultados de estudios similares realizados en población joven, como es el caso de una investigación realizada con pacientes jóvenes cubanos con terapia de reemplazo renal, mostrando un bajo nivel de conocimientos sobre la enfermedad ${ }^{15}$. De igual modo un estudio en Perú realizado con pacientes que padecen diabetes mellitus e hipertensión arterial, revela un desconocimiento sobre la prevención de la enfermedad renal referido a alimentación, actividad física, descanso y control terapéutico ${ }^{17}$.

Así, un estudio en jóvenes de la Universidad de Anáhuac, México ${ }^{21}$ evidencia que la educación escolar y familiar contribuye a la alfabetización en salud. La adopción de estilos de vida y prácticas saludables están estrechamente relacionados con el conocimiento sobre la enfermedad renal y su prevención, así lo demuestra un estudio en la comunidad de la Isla - Chichigalpa indicando que un conocimiento distorsionado sobre la enfermedad y más aún sobre el tratamiento de ésta, conduce a comportamientos en salud no favorables ${ }^{13}$.

En el caso de la Enfermedad Renal Crónica, un estudio en población joven de la Universidad de Málaga (España) durante el curso 2016-2017, con similitud metodo- 
lógica al nuestro, revela también un bajo conocimiento de la misma ${ }^{10}$. Con respecto al conocimiento previo sobre la enfermedad renal, en nuestro estudio hemos encontrado que el $55,7 \%$ no posee ningún tipo de experiencia previa, datos inferiores a los encontrados por los investigadores malagueños ${ }^{10}$. En los resultados de nuestra investigación no se observan diferencias significativas entre hombre y mujeres, de acuerdo con las categorías temáticas, lo que concuerda con estudios previos $^{10,23}$.

Diversas investigaciones coinciden en que el conocimiento de la ERC acerca de la etiología y manifestaciones clínicas es deficiente, tanto en población sana como en población debutante con ERC ${ }^{13-17,24}$. En tanto, los jóvenes pueden conocer las características más básicas de la patología, pero desconocen aquellos aspectos específicos de la misma que permiten adoptar medidas para prevenir su aparición en un futuro ${ }^{10}$.

A partir de los datos obtenidos podemos afirmar que la población universitaria cubana posee un escaso conocimiento sobre la enfermedad renal crónica, encontrando diferencias de acuerdo al año académico y áreas docentes. Estos datos demuestran la necesidad de alfabetizar en temas de salud a la población joven, con el objetivo de prevenir riesgos de enfermedad renal.

Recibido: 12-05-20

Revisado: 22-06-20

Modificado: $30-07-20$

Aceptado: 05-08-20

\section{Bibliografía}

1. Candelaria -Brito JC, Gutiérrez - Gutiérrez C, Bayarre -Vea H D, Acosta -Cruz C, Montes de Oca D M, Labrador - Mazón 0. Caracterización de la enfermedad renal crónica en adultos mayores. Rev. Colomb. Nefrol. 2018;5(2):166-78. [Consultado 24 mar 2020] Disponible en: https://revistanefrologia. org/index.php/rcn/article/view/308/pdf.
2. Pérez-Oliva J, Herrera R, Almaguer M. ¿Cómo mejorar el manejo de la enfermedad renal crónica? Consideraciones y recomendaciones prácticas. Rev haban cienc méd 2008;7(1):1-22. [Consultado 24 de marzo] Disponible en: http://scielo.sld.cu/pdf/ rhcm/v7nl/rhcm09108.pdf.

3. Bedia A. Estilo de vida de los adolescentes y jóvenes y el impacto de la enfermería. [Trabajo fin de grado]. Universidad de Cantabria. 2016. [Consultado 24 mar 2020] Disponible en: https://repositorio.unican.es/xmlui/bitstream/handle/10902/8915/ Bedia\%20Herrero\%20A..pdf?sequence $=4$.

4. Mávita CJ. Alfabetización en salud de una comunidad universitaria del noroeste de México en el año 2016. Inv Ed Med 2018;7(25):36-45.

5. Basagoiti I. Alfabetización en salud. De la información a la acción [pdf]. Valencia: ITACA/TSB; 2012. [Consultado 17 mar 2020] Disponible en: http:// www.salupedia.org/alfabetizacion/

6. Costa-Requena G, Moreso F, Cantarell MC, Serón D. Alfabetización en salud y enfermedad renal crónica. Nefrología. 2017;37(2):115-7.

7. Aparicio Sigueñas KJ. Estilos de vida en pacientes con enfermedad renal crónica en terapia de hemodiálisis en una clínica particular de Lima Metropolitana. [Tesis Doctoral]. Universidad Nacional Mayor de San Marcos, Facultad de Medicina. 2018. [Consultado 24 mar 2020] Disponible en: http://repositorio.unap.edu.pe/bitstream/handle/UNAP/912l/ Colque_Amesquita_Xiomara_Noemi.pdf?sequen$\mathrm{ce}=1$ \&isAllowed=y.

8. Barón $A M$, García Pena AA, García P, Salazar E, Blanco CA, Batancour MC, Amaya N. Factores de riesgo asociados al desarrollo de eventos cardiovasculares en pacientes postrasplante renal. Rev Colomb Cardiol 2017;25(1):13-8.

9. Intra MV, Roales-Nieto J, Moreno San Pedro E. Cambio en las conductas de riesgo y salud en estudiantes universitarios argentinos a lo largo del periodo educativo. Int $\mathrm{J}$ Psychol Psychol. Ther. 2011;11(1):139-47.

10. Sáenz Martínez S, Pérez López F, Martí García C. Conocimiento sobre la enfermedad renal crónica en la población universitaria de Málaga. Enferm Nefrol 2019;22(2):186-93.

11. Muñiz J, Elosua P, Hambleton RK. Directrices para la traducción y adaptación de los tests: segunda edición. Psicothema. 2013;25(2):151-7. 
12. Asociación Americana de Psicología. Manual de Publicaciones de la APA: Editorial EI Manual Moderno. 2010. [Consultado 25 jun 2020] Disponible en: https://psicologia.iztacala.unam.mx/ManualTitulacionPSI/20nov_Libro_ManualdePublicacionesAPA.pdf.

13. Burke M, Kapojos JJ, Sammartino C, Gray NA. Kidney disease health literacy among new patients referred to a nephrology clinic. Intern Med J. 2014; 44(11):1080-6.

14. Chow WL, Joshi VD, Tin AS. Limited knowledge of chronic kidney disease among primary care patients - a cross-sectional survey. BMC Nephrology. 2012;13:54.

15. Domínguez M, Pacheco M, Montero B, Rodríguez $\mathrm{M}$, Rodríguez J. Conocimientos sobre la enfermedad renal crónica y el proceder de hemodiálisis. Rev Ciencias Médicas 2014;18(3):463-72.

16. Gray NA, Kapojos JJ, Burke MT, Sammartino C, Clark CJ. Patient kidney disease knowledge remains inadequate with standard nephrology outpatient care. Clin Kidney J 2016;9(1):113-8.

17. Ulloa Haro ME. Conocimiento sobre la prevención de la enfermedad renal crónica en pacientes con diabetes mellitus e hipertensión arterial en la Clínica Vista Alegre. Lima: Universidad Nacional Mayor de San Marcos; 2016. [Consultado 4 mar 2020] Disponible en: https://cybertesis.unmsm.edu.pe/ handle/20.500.12672/5707.

18. Vicente B, Zerquera J, Rivas $E$, Muñoz J, Gutiérrez $Y$, Castañeda E. Nivel de conocimientos sobre diabetes mellitus en pacientes con diabetes tipo 2 . MediSur. 2010;8(6):21-7.
19. López $\mathrm{P}$, Rejón J, Escobar D, Roblero S, Dávila M, Mandujano Z. Conocimientos nutricionales en estudiantes universitarios del sector público del Estado de Chiapas, México. Inv Ed Med.2017;6(24):22833.

20. Mejía Gonzales CM. Nivel de conocimientos sobre enfermedades de transmisión sexual en gestantes adolescentes del Hospital Carlos Lanfranco La Hoz de Puente Piedra. San Juan Bautista. 2017. [Consultado 8 mar 2020] Disponible en: http://reposito rio.upsjb.edu.pe/handle/upsjb/1213.

21. Konstat-Korzenny E, Fonseca-Portilla R, Majzner-Aronovich S, Lamas- Magallón P. Conocimiento sobre la diabetes mellitus tipo 2 en estudiantes universitarios: Un estudio transversal y observacional en la Universidad Anáhuac México Campus Norte en la Ciudad de México. Rev. chil. endocrinol. Diabetes. 2018;11(4):156-60.

22. Mendicino D, Cuaglini J, Giraldez E, Nepote M. Conocimientos sobre la enfermedad de Chagas en estudiantes de Enfermería de Argentina. Educ Med Super 2019;33(1): el538. [Consultado 8 mar 2020] Disponible en: https://www.medigraphic. com/pdfs/educacion/cem-2019/cem191y.pdf.

23. Carrero J, Hecking M, Chesnaye N, Jager K. Sex and gender disparities in the epidemiology and outcomes of chronic kidney disease. Nat Rev Nephrology. 2017;14(3):151-64.

24. Berríos $A$, Kulakova 0, Jirón A, Martínez M, Martínez J, Urcuyo N. Conocimiento, actitudes y prácticas, sobre enfermedad renal crónica en habitantes de la Comunidad "La Isla" del municipio de Chichigalpa. UNIVERSITAS. 2013;4(1):53-7.

Este artículo se distribuye bajo una Licencia Creative Commons Atribución-NoComercial 4.0 Internacional. https://creativecommons.org/licenses/by-nc/4.0/

Open Access (C) (1) (8) 